\title{
Using Chlorine Atoms to Fine-Tune the Intermolecular Packing and Energy Levels of Efficient Nonfullerene Acceptors
}

Hanjian Lai ${ }^{\dagger,}$, Hui Chen ${ }^{\dagger, \S}$, Yue Shen ${ }^{\perp}$, Meijing Wang ${ }^{\dagger}$, Pengjie Chao ${ }^{\dagger}$, Weicheng Xie ${ }^{\dagger}$, Jianfei $\mathrm{Qu}^{\dagger}$, Bing Yang ${ }^{\perp}$, and Feng $\mathrm{He}^{\dagger, * *}$

†Shenzhen Grubbs Institute, Department of Chemistry and ${ }^{\S}$ Academy for Advanced Interdisciplinary Studies, Southern University of Science and Technology, Shenzhen 518055, China

${ }^{\ddagger}$ School of Chemistry and Chemical Engineering, Harbin Institute of Technology, Harbin 150001, China

${ }^{\perp}$ State Key Lab of Supramolecular Structure and Materials, Jilin University, 2699 Qianjin Avenue, Changchun 130012, China

\section{Corresponding Authors}

*E-mail: hef@sustech.edu.cn (F.H.) 


\section{GENERAL METHODS}

Instruments. ${ }^{1} \mathrm{H}$ NMR spectra were recorded on Bruker AV $400 \mathrm{MHz}$ spectrometer in $\mathrm{CDCl}_{3}$ with tetramethylsilane (TMS) as an internal standard. The MALDI-TOF-MS experiments were recorded on Bruker Autoflex ${ }^{\mathrm{TM}}$ Speed. Preparative gel permeation chromatography purification was performed with a JAI LC-9104 recycling preparative high performance liquid chromatography, and the eluent was chloroform. Cyclic voltammetry (CV) measurements were performed on a CHI 660E potentiostat/galvanostat (Shanghai Chenhua Instrumental Co., Ltd. China) to determine the HOMO and LUMO levels of the polymers, in an acetonitrile solution of $0.1 \mathrm{~mol} \cdot \mathrm{L}^{-1}$ tetrabutylammonium hexafluorophosphate $\left(\left[n-\mathrm{Bu}_{4} \mathrm{~N}\right]^{+}\left[\mathrm{PF}_{6}\right]^{-}\right)$at a potential scan rate of $100 \mathrm{mV} \mathrm{s}^{-1}$ with an $\mathrm{Ag} / \mathrm{Ag}^{+}$reference electrode and a platinum wire counter electrode under a argon atmosphere. Solution and film UV-Vis absorption spectra absorption spectra were recorded on a Shimadzu UV3600 spectrometer. Atom force microscopy (AFM) images were taken on a NanoScopeIIIa controller (Veeco Metrology Group/Digital Instruments, Sant a Barbara, CA), using built-in software (version V6.13R1) to capture images. Transmission electron microscopy (TEM) images were acquired using a HITACHI H-7650 electron microscope operating at an acceleration voltage of $100 \mathrm{kV}$. 
Fabrication and Characterization of OSCs. The fabrication and measurement methods of OSCs devices are as follows: After a thorough cleaning of the indium-tin oxide (ITO)-coated glass substrate with detergent, deionized water, acetone, and isopropyl alcohol under ultrasonication for 15 minutes each and subsequently dried in an oven for 1 minute at $80{ }^{\circ} \mathrm{C}$ under vacuum. The ITO glass substrates were treated with UV-ozone for 15 minutes and then the sol-gel-derived $\mathrm{ZnO}$ films were spin-coated onto the ITO substrates followed by thermal treatment at $200{ }^{\circ} \mathrm{C}$ for $30 \mathrm{~min}$. The total concentration of the PBDB-TF: Acceptor $(1: 1)$ blend solution for spin-coating was 20 $\mathrm{mg} \mathrm{mL} \mathrm{m}^{-1}$ with chlorobenzene as the processing solvent. The additive, 1, 8-diiodooctane (DIO) was added into solution 30 minutes before the spin-coating process. The blend was stirred at room temperature in the glove box overnight. The active layer was spincoating at $2500 \mathrm{rpm}$ for $45 \mathrm{~s}$ to get neat film. A $10 \mathrm{~nm} \mathrm{MoO}$ layer and a $100 \mathrm{~nm} \mathrm{Ag}$ layer were subsequently evaporated through a shadow mask to define the active area of the devices. The integrated device structure is ITO/ZnO/PBDB-TF: Acceptors $/ \mathrm{MoO}_{3} / \mathrm{Ag}$. A solar simulator (Enlitech.Inc) with an AM 1.5G filter was used as a light source to produce an intensity of $100 \mathrm{~mW} \mathrm{~cm}^{-2}$ for the illumination of the photovoltaic cells. The light intensity was calibrated by a $2 \mathrm{~cm} \times 2 \mathrm{~cm}$ calibrated silicon solar cell with KG-3 visible color filter. A shadow mask with a single aperture (4.15 $\mathrm{mm}^{2}$ ) was placed onto the devices in order to accurately define the photoactive area. Steady-state current-voltage $(J-V)$ curves were measured by a Keithley 2400 sourcemeasurement unit under AM 1.5 G spectrum from a solar simulator (Enlitech.Inc) 
calibrated by a silicon reference cell (Hamamatsu S1133 color, with KG-5 visible fith). The relationship of $J_{\mathrm{sc}}$ to the light intensity were measured by steady-state currentvoltage measurement, the light intensity was modulated by neutral density filters (NDF) with different values of optical density (OD). The external quantum efficiency (EQE) was measured by a solar cells-photodetector responsibility measurement system (Enlitech.Inc).

Electron-only and hole-only devices fabrication. Electron-only devices were fabricated with the device structure of ITO/ZnO/PBDB-TF: Acceptors/PDINO/Al, while the hole-only devices were fabricated with the device structure of ITO/PEDOT: $\mathrm{PSS} / \mathrm{blends} / \mathrm{MoO}_{3} / \mathrm{Ag}$. The mobilities were determined by fitting the dark current to the model of a single carrier SCLC, ${ }^{1,2}$ which is described by the equation:

$$
J=\frac{9}{8} \varepsilon_{0} \varepsilon_{r} \mu_{h} \frac{V^{2}}{d^{3}}
$$

where $J$ is the current, $\mu_{\mathrm{h}}$ is the zero-field mobility, $\varepsilon_{0}$ is the permittivity of free space, $\varepsilon_{\mathrm{r}}$ is the relative permittivity of the material, $d$ is the thickness of the active layer, and $V$ is the effective voltage. 


\section{EXPERIMENTAL SECTION}

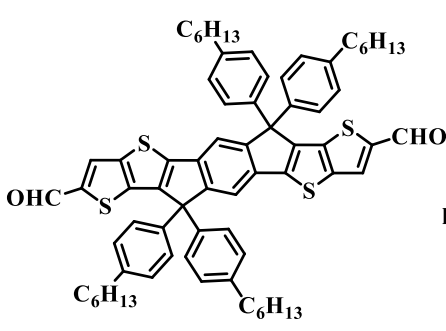

IDTT-CHO
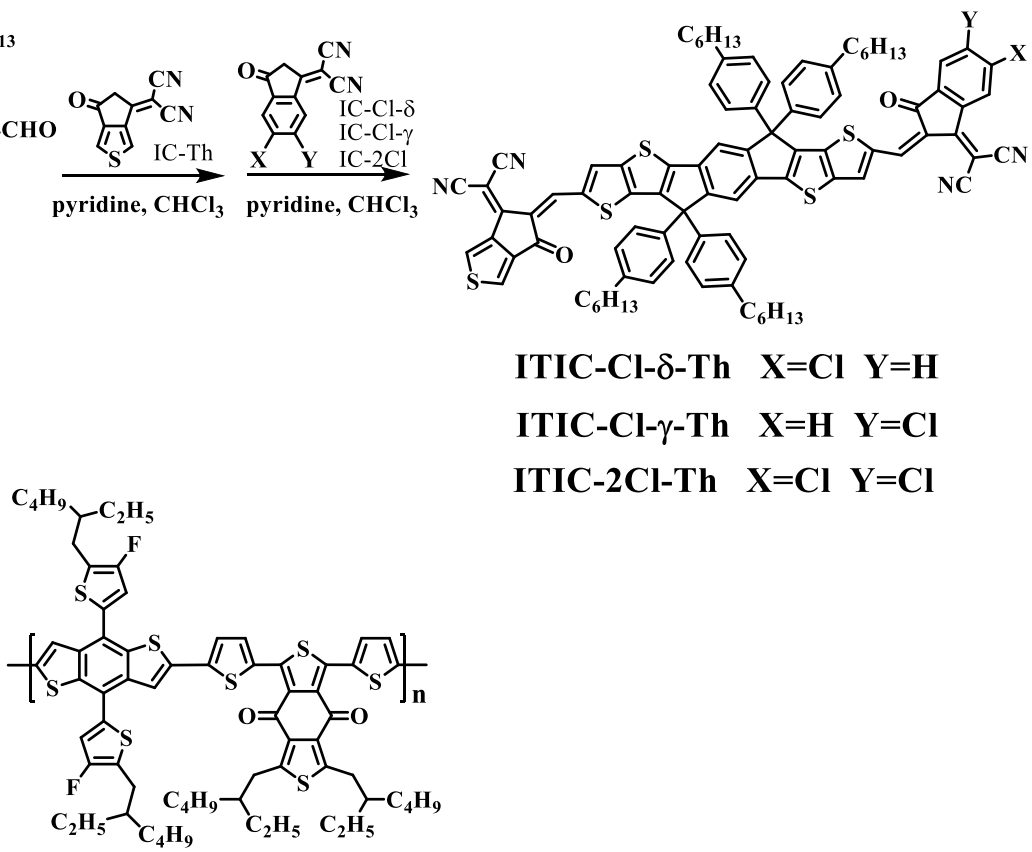

ITIC-Cl- $\delta$-Th $\mathrm{X}=\mathrm{Cl} \mathrm{Y}=\mathrm{H}$

ITIC-Cl- $\gamma-$ Th $\mathrm{X}=\mathrm{H} \quad \mathrm{Y}=\mathrm{Cl}$

ITIC-2Cl-Th $\mathrm{X}=\mathrm{Cl} \mathrm{Y}=\mathrm{Cl}$

PBDB-TF

Scheme S1. The synthetic routes of ITIC-Cl- $\boldsymbol{\delta}$-Th, ITIC-Cl- $\boldsymbol{\gamma}$-Th, ITIC-2Cl-Th and the structure of PBDB-TF.

Materials: Compound IDTT-CHO, IC-2Cl, IC-Th, IC-Cl- $\boldsymbol{\gamma}$ and IC-Cl- $\boldsymbol{\delta}$ were synthesized according previously reported approaches. ${ }^{3-5}$ All the other chemicals were purchased as reagent grade from J\&K, Energy, Macklin, and Sigma-Aldrich, and used without further purification. All solvents for reactions were freshly distilled immediately prior to use.

Compound ITIC-Cl- $\boldsymbol{\delta}$-Th: A mixture of IDTT-CHO (200 mg, $0.186 \mathrm{mmol}$ ), IC-Th (38 mg, $0.186 \mathrm{mmol})$ in chloroform/pyridine $(30 \mathrm{ml} / 0.5 \mathrm{ml})$ was refluxed 12 hours under Argon, then the IC-Cl- $\boldsymbol{\delta}$ (43 $\mathrm{mg}, 0.186 \mathrm{mmol}$ ) was added for further 12 hours. 
The reaction mixture was then cooled to room temperature and then extracted with chloroform. The organic layer was washed with brine and dried over $\mathrm{MgSO}_{4}$. The crude product was purified by flash column chromatography with chloroform as eluent and further purified with cycling preparative HPLC to get the product (100 mg, 36.6\%). ${ }^{1} \mathrm{H}$ NMR (400 MHz, $\left.\mathrm{CDCl}_{3}\right) \delta: 8.87(\mathrm{~s}, 1 \mathrm{H}), 8.80(\mathrm{~s}, 1 \mathrm{H}), 8.60-8.63(\mathrm{~d}, 1 \mathrm{H}), 8.38(\mathrm{~s}, 1 \mathrm{H})$, 8.18(s, 1H), $8.24(\mathrm{~s}, 1 \mathrm{H}), 7.92(\mathrm{~s}, 1 \mathrm{H}), 7.85(\mathrm{~s}, 1 \mathrm{H}), 7.69-7.71(\mathrm{~d}, 1 \mathrm{H}), 7.64(\mathrm{~s}, 2 \mathrm{H})$, 7.13-7.22(m, 16H), 2.55-2.59(t, 8H), 1.57-1.61(m, 8H), 1.26-1.36(m, 24H), 0.840.87(m, 12H). MALDI-TOF-MS calcd for C94H80Cl2N4O2S4 (M+): 1466.4495, found: 1471.5451 .

Compound ITIC-Cl- $\boldsymbol{\gamma}$-Th: A mixture of IDTT-CHO (200 mg, $0.186 \mathrm{mmol})$, IC-Th (38 mg, $0.186 \mathrm{mmol})$ in chloroform/pyridine $(30 \mathrm{ml} / 0.5 \mathrm{ml})$ was refluxed 12 hours under Argon, then the IC-Cl- $\gamma$ (43 mg, $0.186 \mathrm{mmol}$ ) was added for further 12 hours. The reaction mixture was then cooled to room temperature and then extracted with chloroform. The organic layer was washed with brine and dried over $\mathrm{MgSO}_{4}$. The crude product was purified by flash column chromatography with chloroform as eluent and further purified with cycling preparative HPLC to get the product $(110 \mathrm{mg}, 40.2 \%) .{ }^{1} \mathrm{H}$ NMR (400 MHz, $\left.\mathrm{CDCl}_{3}\right) \delta: 8.87(\mathrm{~s}, 1 \mathrm{H}), 8.80(\mathrm{~s}, 1 \mathrm{H}), 8.65(\mathrm{~s}, 1 \mathrm{H}), 8.37-8.38(\mathrm{~m}, 1 \mathrm{H})$, 8.18-8.22(d, 2H), 7.92-7.93 (m, 1H), 7.83-7.85 (d, 1H), 7.68-7.70 (d, 1H), 7.63-7.64 (m, 2H), 7.13-7.22(m, 16H), 2.54-2.58(t, 8H), 1.57-1.63(m, 8H), 1.26-1.45(m, 24H), 0.84-0.87(m, 12H). MALDI-TOF-MS calcd for C94H80Cl2N4O2S4 (M+): 1466.4495, found: 1469.5517 . 
Compound ITIC-2Cl-Th: A mixture of IDTT-CHO (200 mg, $0.186 \mathrm{mmol})$, IC-Th (38 $\mathrm{mg}, 0.186 \mathrm{mmol})$ in chloroform/pyridine $(30 \mathrm{ml} / 0.5 \mathrm{ml})$ was refluxed 12 hours under Argon, then the IC-2Cl (50 mg, $0.186 \mathrm{mmol})$ was added for further 12 hours. The reaction mixture was then cooled to room temperature and then extracted with chloroform. The organic layer was washed with brine and dried over $\mathrm{MgSO}_{4}$. The crude product was purified by flash column chromatography with chloroform as eluent and further purified with cycling preparative HPLC to get the product (110 mg, 39.4\%). ${ }^{1} \mathrm{H}$ NMR (400 MHz, $\left.\mathrm{CDCl}_{3}\right) \delta: 8.87(\mathrm{~s}, 1 \mathrm{H}), 8.80(\mathrm{~s}, 1 \mathrm{H}), 8.76(\mathrm{~s}, 1 \mathrm{H}), 8.38(\mathrm{~d}, 1 \mathrm{H}), 8.24$ (s, 1H), $8.18(\mathrm{~s}, 1 \mathrm{H}), 7.95(\mathrm{~s}, 1 \mathrm{H}), 7.93-7.94(\mathrm{~d}, 1 \mathrm{H}), 7.64(\mathrm{~s}, 2 \mathrm{H}), 7.13-7.22(\mathrm{~m}, 16 \mathrm{H})$, $2.55-2.59(\mathrm{t}, 8 \mathrm{H}), 1.57-1.61(\mathrm{~m}, 8 \mathrm{H}), 1.26-1.33(\mathrm{~m}, 24 \mathrm{H}), 0.84-0.87(\mathrm{~m}, 12 \mathrm{H})$. MALDITOF-MS calcd for C94H80Cl2N4O2S4 (M+): 1500.4105, found: 1503.5431. 

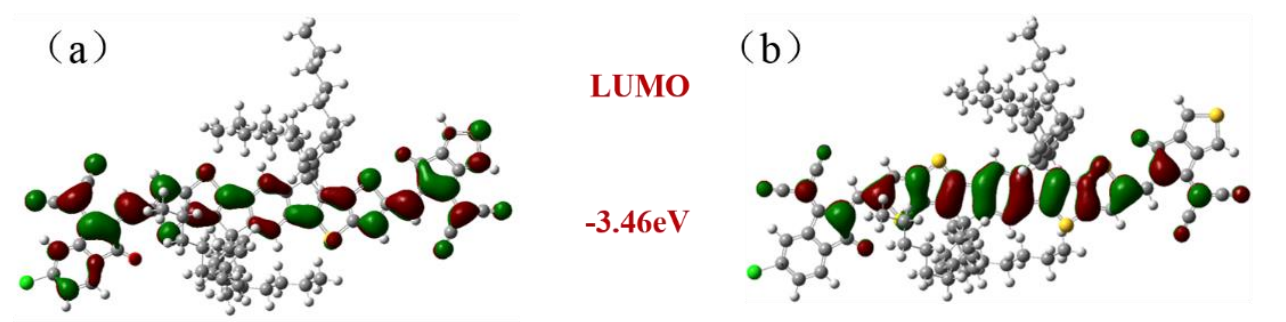

HOMO
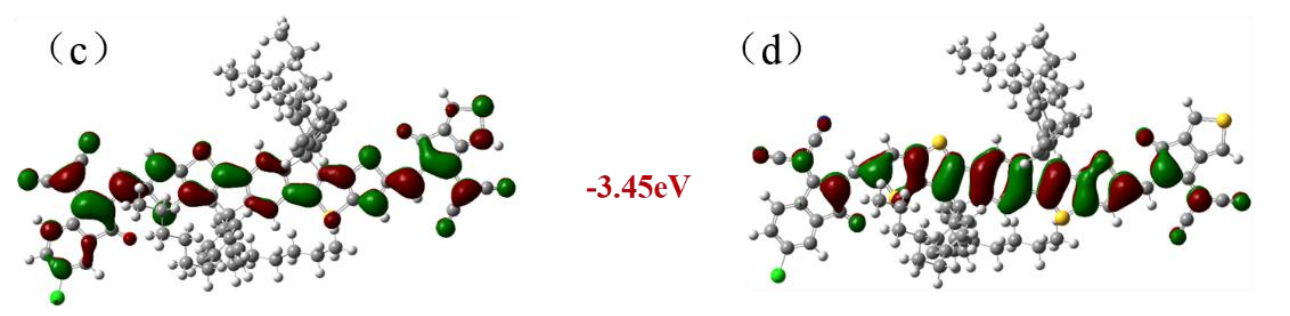

$-5.54 \mathrm{eV}$
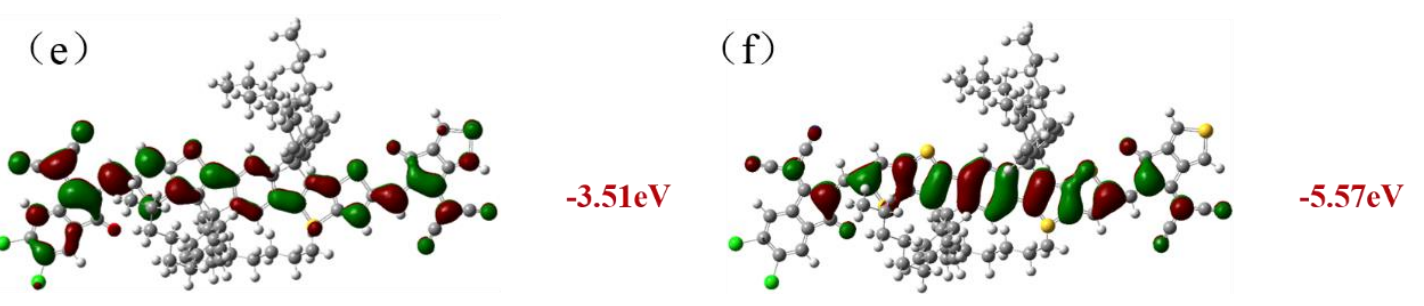

Figure S1. The theoretical calculation energy levels of ITIC-Cl- $\boldsymbol{\delta}-\mathbf{T h}(\mathrm{a}, \mathrm{b})$, ITIC-Cl$\gamma$-Th (c, d) and ITIC-2Cl-Th (e, f). 


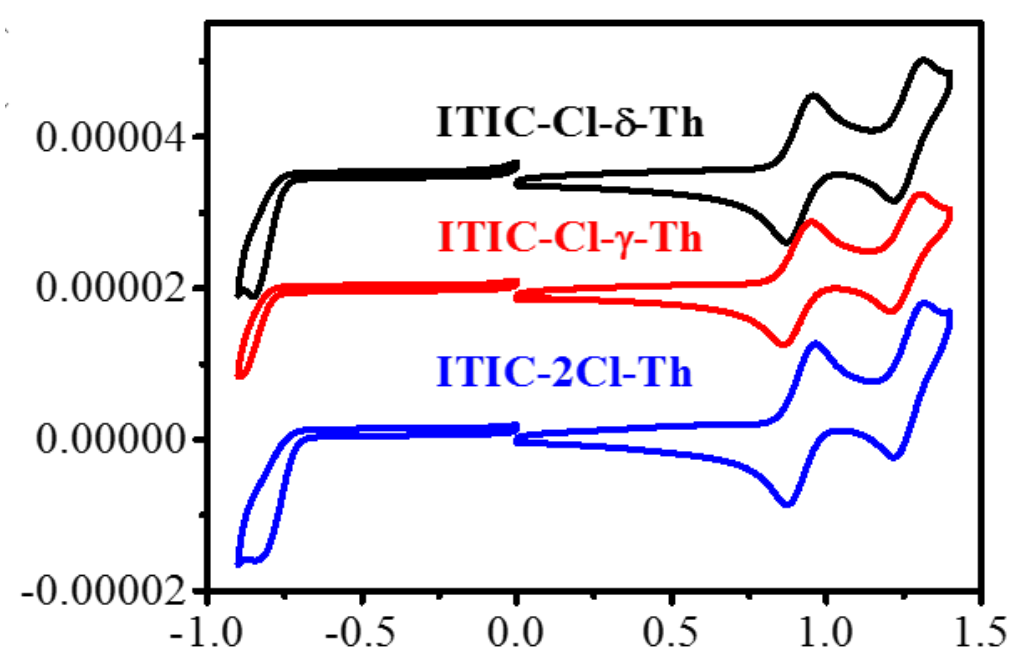

Figure S2. Cyclic voltammograms of three molecules in dichloromethane with $(n-$ $\mathrm{Bu})_{4} \mathrm{NPF}_{6}(0.1 \mathrm{M})$ as supporting electrolyte, Pt wire as counter electrode, and $\mathrm{Ag} / \mathrm{Ag}^{+}$ as reference electrode.
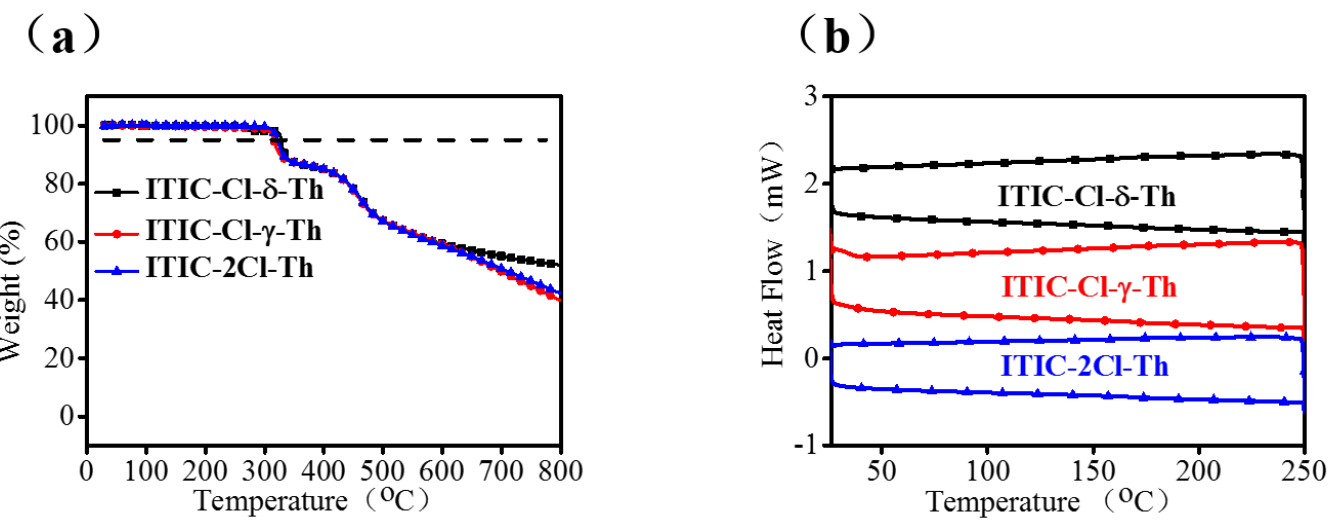

Figure S3. (a) Double logarithmic plots of the current density $(J)$ versus voltage $(V)$ curves for the hole-only, (b) electron-only of three acceptors-based devices. 


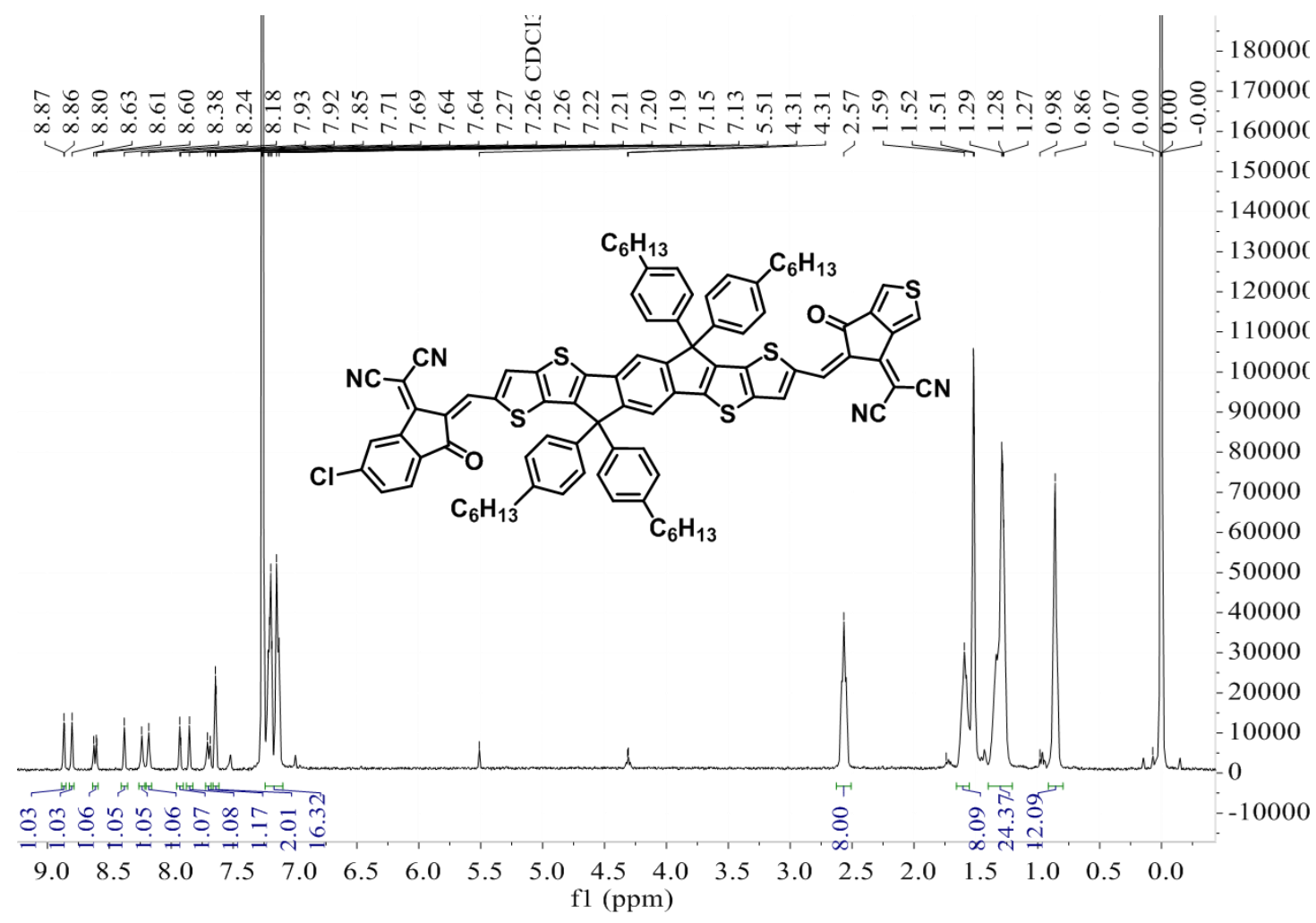

Figure S4. ${ }^{1} \mathrm{H}$ NMR of ITIC-Cl- $\boldsymbol{\delta}-\mathbf{T h}$ in $\mathrm{CDCl}_{3}$.

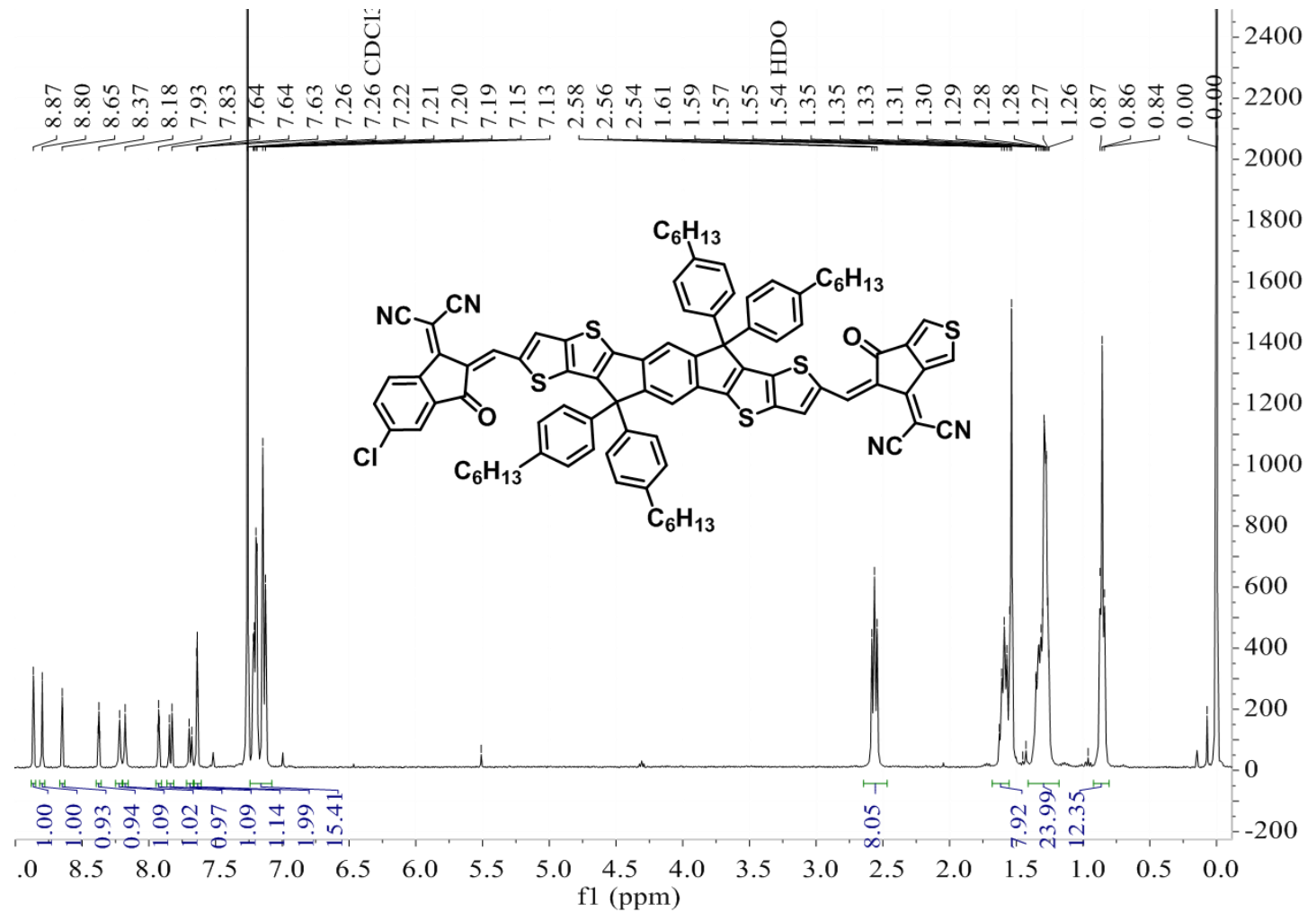

Figure S5. ${ }^{1} \mathrm{H}$ NMR of ITIC-Cl- $\boldsymbol{\gamma}$-Th in $\mathrm{CDCl}_{3}$. 


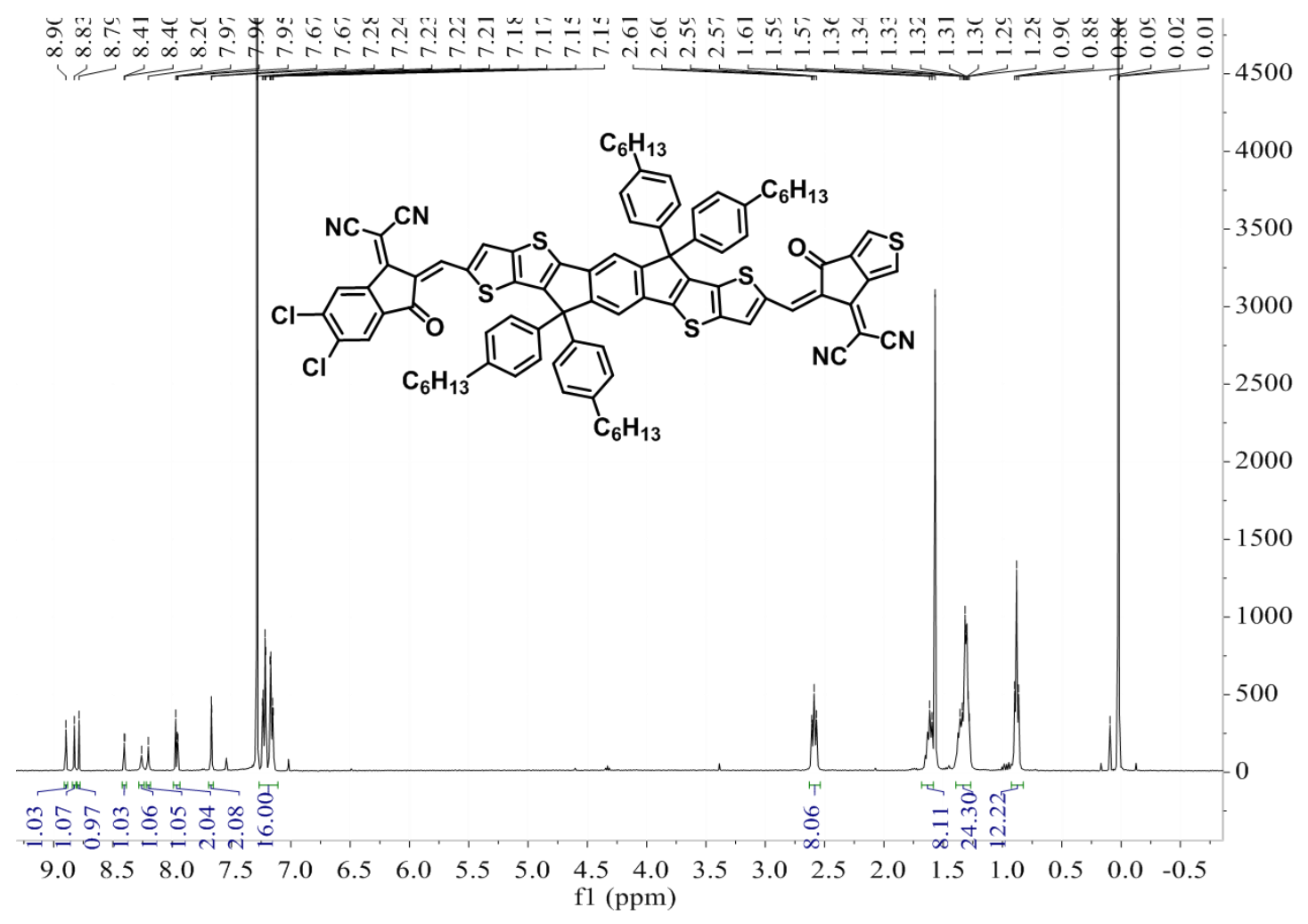

Figure S6. ${ }^{1} \mathrm{H}$ NMR of ITIC-2Cl-Th in $\mathrm{CDCl}_{3}$. 


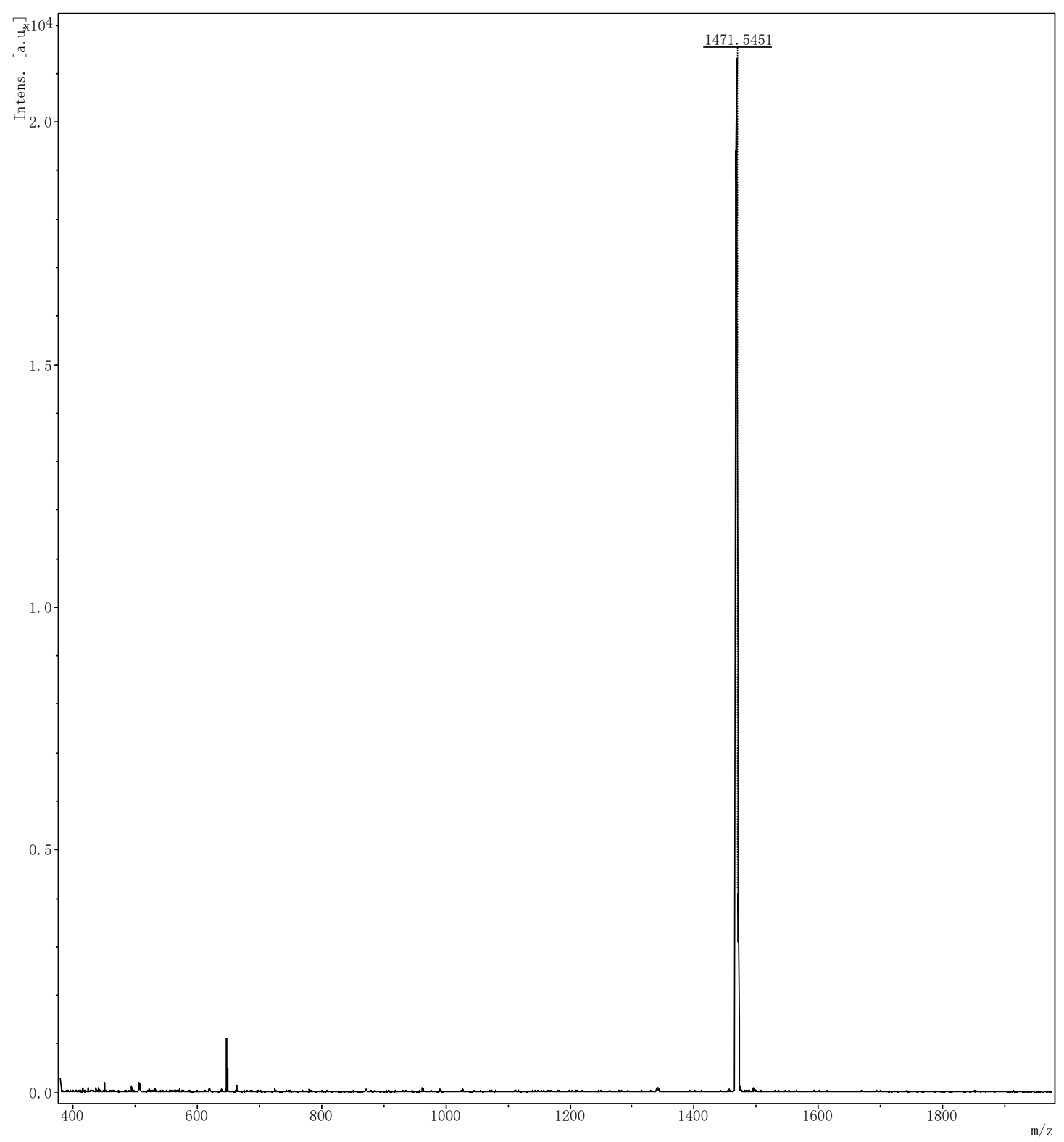

Figure S7. MALDI-TOF-MS spectrum of ITIC-Cl- $\boldsymbol{\delta}-\mathbf{T h}$. 


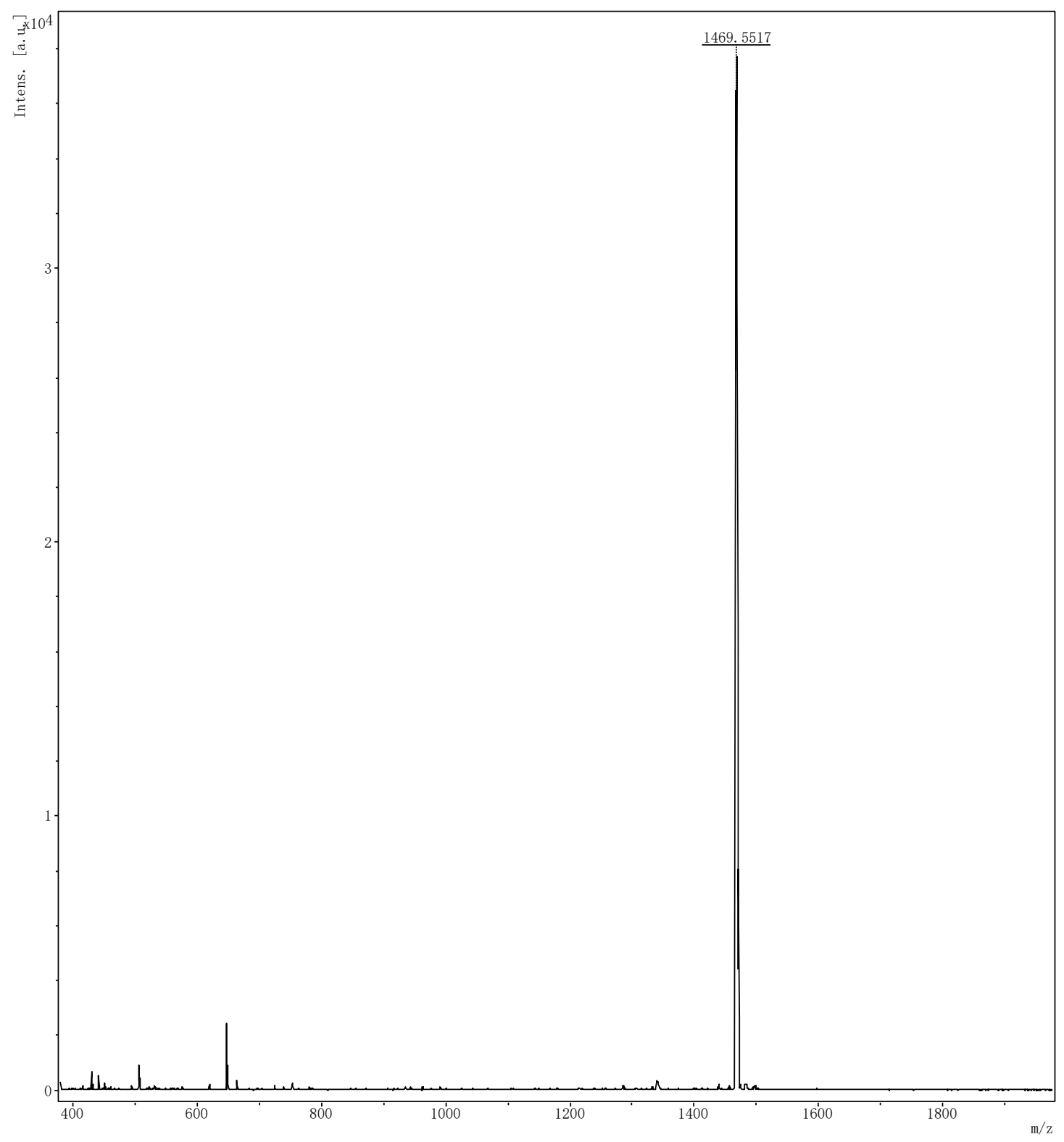

Figure S8. MALDI-TOF-MS spectrum of ITIC-Cl- $\boldsymbol{\gamma}-\mathbf{T h}$. 


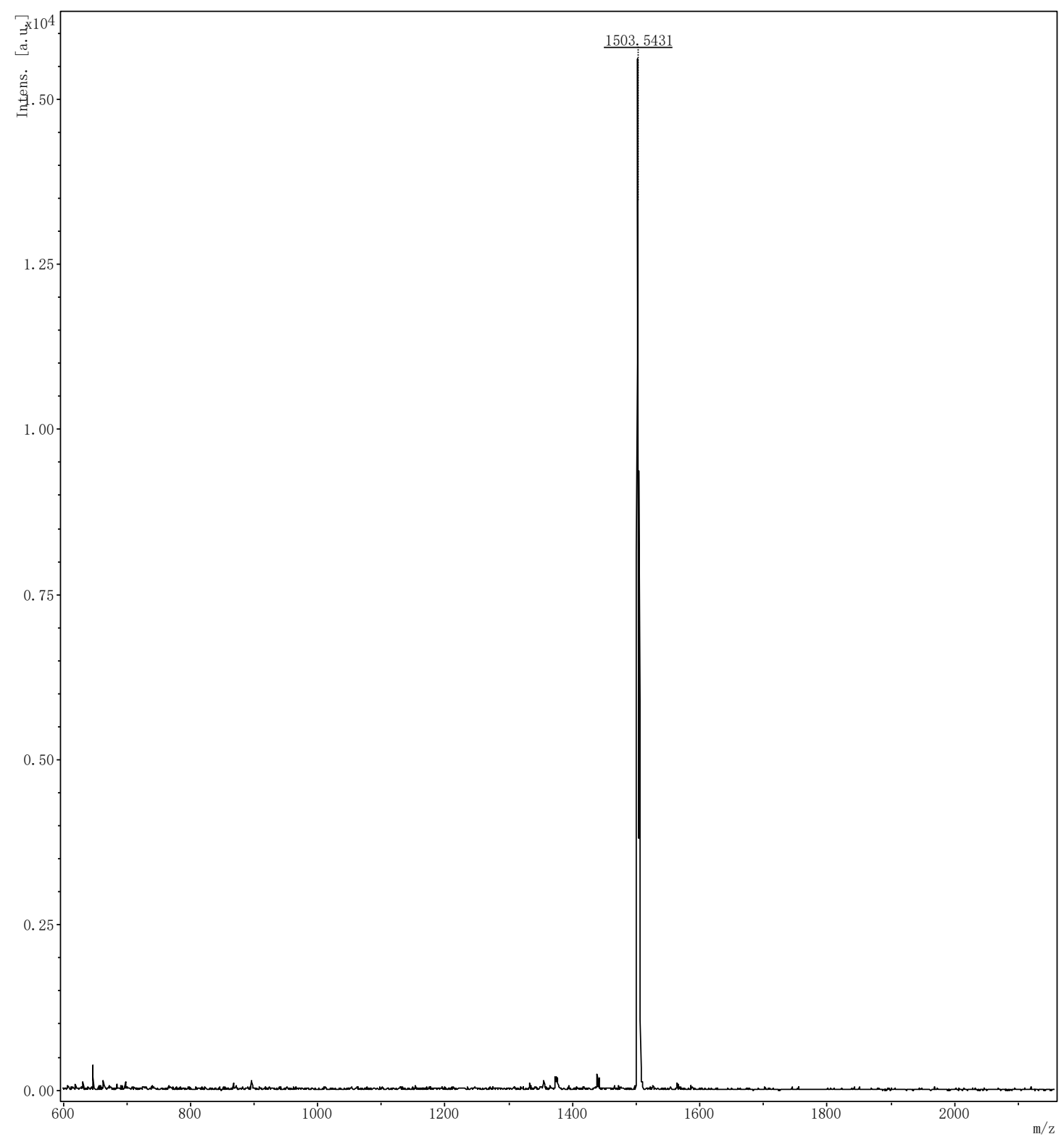

Figure S9. MALDI-TOF-MS spectrum of ITIC-2Cl-Th. 


\section{REFERENCES}

(1) Mihailetchi, V. D.; Wildeman, J.; Blom, P. W. Space-Charge Limited Photocurrent. Phys. Rev. Lett. 2005, 94, 126602-126605.

(2) Davids, P. S.; Campbell, I. H.; Smith, D. L. Device Model for Single Carrier Organic Diodes. J. Appl. Phys. 1997, 82, 6319-6325.

(3) Lin, Y.; Wang, J.; Zhang, Z. G.; Bai, H.; Li, Y.; Zhu, D.; Zhan, X. An Electron Acceptor Challenging Fullerenes for Efficient Polymer Solar Cells. Adv. Mater. 2015, $27,1170-1174$.

(4) Yao, H.; Cui, Y.; Yu, R.; Gao, B.; Zhang, H.; Hou, J. Design, Synthesis, and Photovoltaic Characterization of a Small Molecular Acceptor with an Ultra-Narrow Band Gap. Angew. Chem. Int. Ed. 2017, 56, 3045-3049.

(5) Li, Y.; Lin, J.-D.; Che, X.; Qu, Y.; Liu, F.; Liao, L. S.; Forrest, S. R. High Efficiency Near-Infrared and Semitransparent Non-Fullerene Acceptor Organic Photovoltaic Cells. J. Am. Chem. Soc. 2017, 139, 17114-17119. 